\title{
Comparison between a symmetric bidirectional-pumping and a unidirectional-pumping configurations in an erbium fiber ring laser
}

\author{
M.A. Quintela, C. Quintela, M. Lomer, F.J. Madruga, O.M. Conde and J.M. Lopez-Higuera \\ Photonics Engineering Group, Dpto. TEISA, ETSIyT - University of Cantabria, \\ Avda. los Castros s/n, cp. 39005, Santander, Spain
}

\begin{abstract}
An experimental comparative study between two wide-band wavelength-tunable erbium doped fiber ring lasers (EDFRLs) with the same active fiber length but with two different pumping configuration (forward unidirectional pumping and symmetric bidirectional pumping) is reported in this paper. Both fiber lasers cover almost the whole Cband and L-band with a single setup laser. The signal wavelength can be tuned in a wide range of $60 \mathrm{~nm}$ with the two presented lasers. Nevertheless, experimental results verify that a higher output power is obtained with the bidirectional pump configuration.
\end{abstract}

Keywords: Optical fiber ring lasers, erbium doped fiber (EDF), ring lasers, laser tuning

\section{INTRODUCTION}

Erbium-doped fiber lasers (EDFL) have been applied both sensing and telecommunication fields (wavelength-division multiplexing, fiber-optic sensors, optical spectroscopy, etc) due to their potential characteristics. There are basically three types of EDFL: fiber ring laser, distributed Bragg reflector (DBR) fiber laser and distributed feedback (DFB) fiber laser.

The DBR and DFB fiber laser are high-quality laser sources, but nevertheless these present some limitations. The length of the medium gain is usually short $(<10 \mathrm{~cm})$. As a consequence, it is very difficult to achieve the lasing with fibers of low erbium concentration and a self-pulsation can occur due to the effect of erbium ion clustering with fibers of high erbium concentration. This fundamental problem can be overcome by codoping the erbium doped fiber (EDF) with ytterbium [1]. On the other hand, it is not possible to include tunable filter for tuning the laser due to the short length of the active medium.

The erbium doped fiber ring lasers (EDFRL) is a very competitive structure to achieve simultaneously the requirements of many applications. Although the EDF has a low erbium concentration, the EDFRL can easy achieve the lasing by using a very long active fiber $(>10 \mathrm{~m})$. Other important advantage of these lasers is the large range wavelength tunability. Tunable EDFRL can cover the conventional wavelength C-band that matches well to the gain range of the EDF. Furthermore, these lasers can also cover the long wavelength band (L-band) with an appropriate election of the EDF length [2]. The latter is important in optical communication systems, where an increase in the traffic demand requires the incorporation of the L-band. In gas sensing the development of L-band fiber lasers are also very interesting. For example, the methane gas presents a high absorption peak in the L-band [3].

A bigger and spectrally wider amplified spontaneous emission (ASE) is generated inside the EDF, which is an advantage for this laser structure. In addition to our purposes, the ring cavity provides feedback for laser oscillation using the ASE power.

To our knowledge, in almost all published works about EDFRLs the pumping configuration used is unidirectional. The unidirectional pumping launched into the EDF in the direction marked by the isolator (forward unidirectional pump configuration) is the appropriate configuration to implement an L-band EDFRL. However, a high pumping efficiency and a high output power can be obtained using a bidirectional pump configuration [4] [5]. In this paper, an experimental comparative study between two wide-band wavelength-tunable EDFRLs covering the C-band and L-band, with the same length of the active fiber but with two different pumping configurations (forward unidirectional pumping and symmetric bidirectional pumping) is presented. It has been successfully validated in the laboratory.

Third European Workshop on Optical Fibre Sensors, Antonello Cutolo, Brian Culshaw, José Miguel López-Higuera, Eds., Proceedings of SPIE Vol. 6619, 66192O, (2007) $\cdot 0277-786$ X/07/\$18 $\cdot$ doi: 10.1117/12.738538 


\section{EXPERIMENTAL SET-UP}

The schematic setups of the two tunable EDFRLs are shown in Fig. 1. The cavity was composed of a tunable filter, an optical isolator, a polarization controller (PC), a 3dB optical coupler and one or two 1480/1550 nm WDM's depending on the pumping configuration. The tunable filter determines the lasing wavelength of the EDFRL. A grating filter (TB9 JDS Uniphase) with a wide tunable range (>100nm) has been used. An optical isolator ensures unidirectional oscillation around the ring and a polarization controller provides an intracavity mechanism to compensate for the slight polarization dependent gain. The light is coupled out through one port of the optical coupler. In both lasers, the EDF was pumped by a $1480 \mathrm{~nm}$ pump laser (Keopsys Raman fiber laser). In the unidirectional pumping, the pump signal is launched into the active fiber through a WDM. In the bidirectional pumping, the EDF was pumped by the pump laser for both ends of the active fiber through a $3 \mathrm{~dB}$ optical coupler and two WDMs. Therefore, the pump power launched into the two ends of the $\mathrm{EDF}$ is the same (symmetric bidirectional pump configuration).

The gain medium in the cavity was formed by two pieces of $\mathrm{EDF}\left(\mathrm{EDF}_{\mathrm{x}}\right.$ and $\left.\mathrm{EDF}_{\mathrm{y}}\right)$ separated by the optical isolator. The lengths and peak absorption at $1530 \mathrm{~nm}$ of the $\mathrm{EDF}_{\mathrm{x}}$ are $26 \mathrm{~m}$ and $4.4 \mathrm{~dB} / \mathrm{m}$, respectively, and the ones of the $\mathrm{EDF}_{\mathrm{y}}$ are $10 \mathrm{~m}$ and $34.5 \mathrm{~dB} / \mathrm{m}$. In the first section of the $\mathrm{EDF}\left(\mathrm{EDF}_{\mathrm{x}}\right)$ the inversion of erbium ions populations takes place when this fiber is pumped at $1480 \mathrm{~nm}$, so the ASE in the C-band is obtained. The EDF $_{\mathrm{y}}$ utilizes the ASE generated by the $\mathrm{EDF}_{\mathrm{x}}$, including the forward and the backward ASE, as the secondary pump source to obtain L-band ASE [6], and therefore to obtain the desired change in the output signal towards the L-band. In the bidirectional pump configuration, the weak ASE generated by the forward pump is amplified by the C-band ASE generated by the backward pump [5]. This causes an increase in the gain, and as a consequence, a higher output optical power will be obtained according to the unidirectional pump configuration.

In both setups, the isolator is positioned between the $\mathrm{EDF}_{\mathrm{x}}$ and the $\mathrm{EDF}_{\mathrm{y}}$. This one allows obtain a higher output optical power in the band L. This is due to the use of the forward and backward ASE generated in the EDF $_{x}$ to pump the $\mathrm{EDF}_{\mathrm{y}}$. Nevertheless, when the two sections of the EDF are followed and the isolator is placed at any point of the ring between the positions A and B (Fig. 1), the backward ASE generated in the $\mathrm{EDF}_{\mathrm{x}}$ is absorbed by the isolator and loosed. Therefore only the forward ASE generated in the $\mathrm{EDF}_{\mathrm{x}}$ was used to pump the $\mathrm{EDF}_{\mathrm{y}}$.
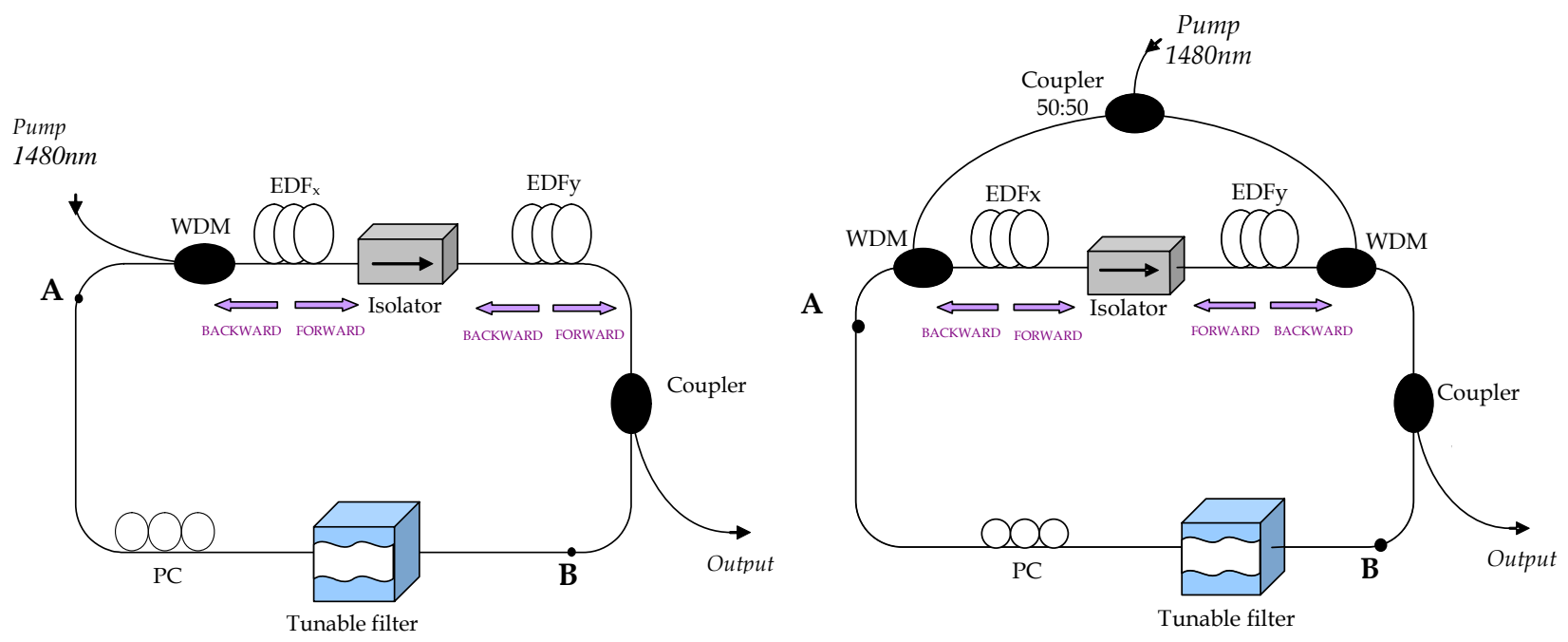

Fig. 1. Experimental setups of the tunable EDFRLs: (a) forward unidirectional pump configuration (b) symmetric bidirectional pump configuration.

\section{EXPERIMENTAL RESULTS}

A sample of the experimental results of the output optical spectrum of both EDFRLs working in different signal wavelengths for a total pump power of $600 \mathrm{~mW}$ at $1480 \mathrm{~nm}$ are shown in Fig. 2(a) and Fig. 2(b). The tuning range of the EDFRL with the unidirectional pump configuration is $60 \mathrm{~nm}(1550 \mathrm{~nm}-1610 \mathrm{~nm})$ (Fig. 2(a)) and the tuning range of the EDFRL with the symmetric bidirectional pump configuration is $60 \mathrm{~nm}(1540 \mathrm{~nm}-1600 \mathrm{~nm})$ (Fig. 2(b)). These fiber lasers 
present the same tuning range, therefore almost the whole C-band and the L-band is covered with the two fiber lasers. Nevertheless, the EDFRL with the bidirectional pumping starts and leaves lasing in inferior wavelengths, i.e., this one cover more C-band and les L-band. This is due to the generated ASE was displaced to the left. It can be also observed that the output optical power in both lasers is not constant in all amplification spectral range for a constant pump power. The output optical power is higher in the spectral regions where the ASE power generated is higher.

Although these EDFRLs have a good signal-to-noise ratio (SNR) in the middle of the tuning range, the noise increases at the edge of that range. The noise could have been eliminated if the optical filter is placed before the output coupler.

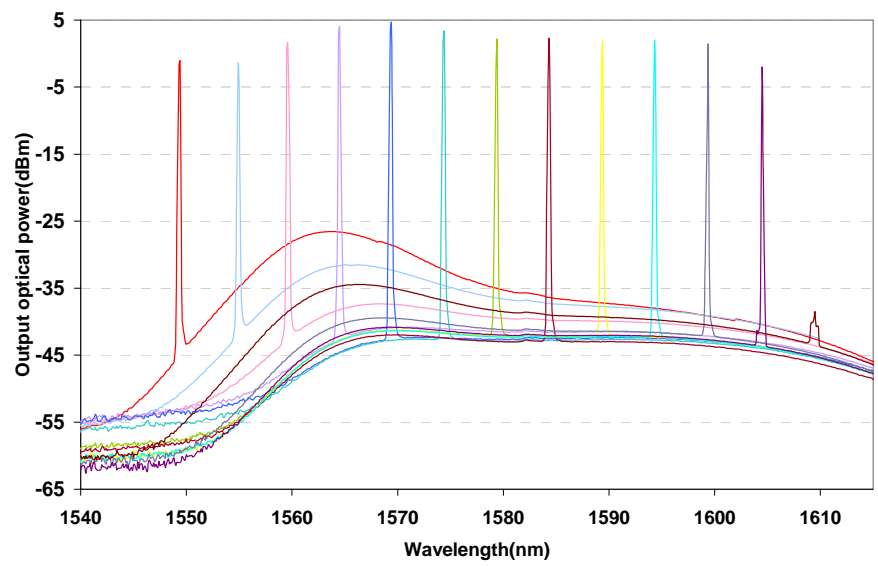

(a)

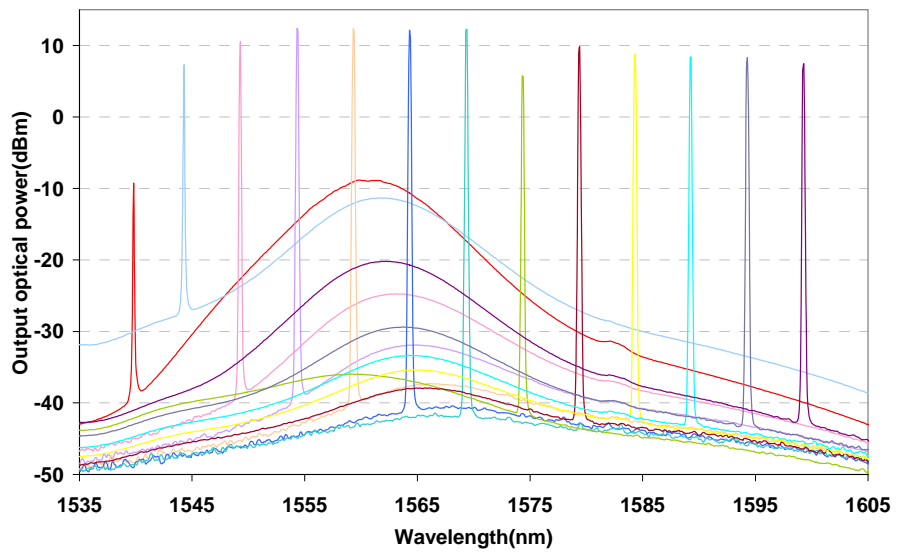

(b)

Fig. 2. Output optical spectrum of the EDFRLs operating in different wavelength for a total pump power of $600 \mathrm{~mW}$ at $1480 \mathrm{~nm}$. (a) Forward unidirectional pump configuration; the tuning range is $60 \mathrm{~nm}$ $(1550 \mathrm{~nm}-1610 \mathrm{~nm})$. (b) Symmetric bidirectional pump configuration; the tuning range is $60 \mathrm{~nm}(1540 \mathrm{~nm}-$ $1600 \mathrm{~nm})$.

Fig.3 (a)-(b) shows the output optical power as a function of the pump power for three signal wavelengths in the two pump configurations (unidirectional forward pumping and bidirectional pumping) with the same total pump power. As expected, for each signal wavelength and all measured pump powers, a higher output optical power is obtained with the bidirectional pumping. For example, for a pump power of $600 \mathrm{~mW}$ and a signal wavelength of $1550 \mathrm{~nm}$ the output optical power is approximately six times higher in the bidirectional pump configuration.

In these experimentally validated EDFRLs, it has been observed that a bidirectional pumping permits achieve a higher output optical power although this implies a reduction in the long wavelength end of the tuning range. As the active length exhibits an influence in the short and long wavelength ends of the tuning range, a possible solution to achieve an EDFRL with a bidirectional pump configuration and a tuning range that covers more L-band, and without reduction of the output power is an increase of the active fiber length. 


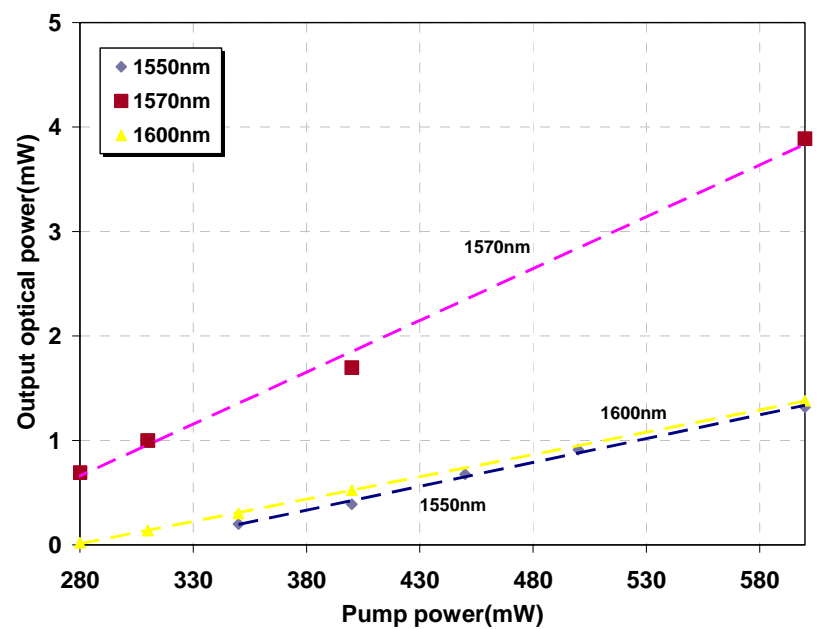

(a)

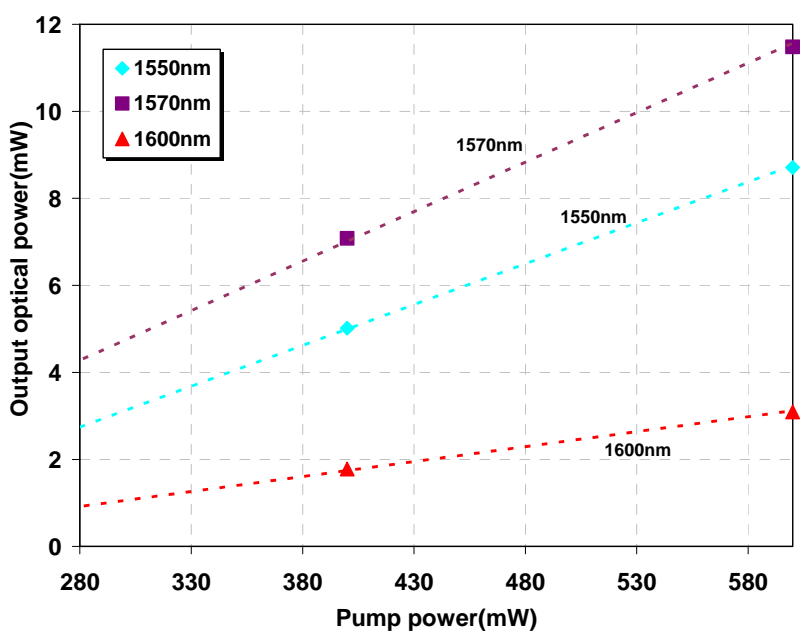

(b)

Fig. 3. Output optical power as a function of the pump power for three signal wavelengths (1550, 1570 and $1600 \mathrm{~nm}$ ) in the two pumping configurations: (a) forward unidirectional pumping and (b) bidirectional pumping.

\section{CONCLUSION}

In this letter two wide-band wavelength-tunable EDFRLs with different pump configurations: forward unidirectional and symmetric bidirectional are reported. From the experimental results, it is found that the bidirectional pump configuration is the optimal to achieve a high output power. A maximum output power of almost $9 \mathrm{~mW}$ at $1550 \mathrm{~nm}$ with a pump power of $600 \mathrm{~mW}$ is obtained, which is approximately six times higher than the one achieved with a unidirectional pumping configuration. Both fiber lasers present a maximum tuning range of $60 \mathrm{~nm}$. Nevertheless, more Cband and less L-band is cover with the bidirectional configuration. A higher active fiber length can give rise to an increase of the short and long wavelength ends of the tuning range. These fiber lasers may be used for gas sensors based on absorption spectroscopy due to theirs characteristics: narrow linewidth and several gar absorption lines fall within the tuning range.

\section{ACKNOWLEDGMENT}

This work was supported in part by the Spanish Government TEC2004-05936-C02 and TEC2005-08218-C02-02 projects. The authors want to thank Pedro Corredera of the CSIC R\&D institution for having supplied the pump power source.

\section{REFERENCES}

1. W.H. Loh, B.N. Samson, L. Dong, G.J. Cowle, K. Hsu, "High performance single frequency fiber grating-based erbium:ytterbium-codoped fiber lasers," Journal of Lightwave Technology, 16(1), 114-118 (1998).

2. X. Dong, P. Shum, N.Q. Ngo, C.C. Chan, B.O. Guan, H.Y. Tam, "Effects of active fiber length on the tunability of erbium-doped fiber ring lasers," Opt. Express, 11(26), 6622-3627 (2003).

3. F.W. Tong, W. Jin, D.N Wang, P.K.A Wai, “A multi-wavelength fiber laser for methane gas detection," Proc. SPIE, 5502, 362365 (2004).

4. S.C. Tsai, T.C Tsai, P.C Law, Y.K. Chen, "High pumping-efficiency L-band erbium-doped fiber ASE source using double-pass bidirectional-pumping configuration," IEEE Photon. Technol. Lett., 15(2), 197-199 (2003).

5. W. Huang, X. Wang, Z. Cai, H. Xu, C Ye, “A pump power insensitive high stability L-band erbium-doped superfluorescent fiber source," J. Opt. A: Pure Appl. Opt., 7(4), 179-183 (2005).

6. S. Hsu, T. Liang, Y. Chen,_"Optimum configuration and design of L-band erbium-doped superfluorescent fiber source,” Jpn J. Appl. Phys, 41, 3724-3729 (2002). 